\title{
Towards Automated Condition Monitoring of Blowout Preventer Wellbore Packers
}

\author{
Se Un Park ${ }^{1}$, Rajesh Kumar Bade ${ }^{2}$, Daniel Barker ${ }^{3}$, and Daniel Edgardo Viassolo ${ }^{4}$ \\ 1,4Schlumberger, 1430 Enclave Pkwy., Houston, TX, 77077, USA \\ spark4@slb.com,dviassolo@slb.com
}

2,3Schlumberger, 4601 Westway Park Blvd., Houston, TX 77041, USA

rbade@cameron.slb.com,dbarker3@cameron.slb.com

\begin{abstract}
According to Bureau of Safety and Environmental Enforcement (BSEE), in 2017 a total of 45 out of 59 rigs operating in the Gulf of Mexico reported component failures of well control related equipment. The aftermath of the oil spill from the Deepwater Horizon rig provides stark illustration that acceptance of the equipment failure status quo is untenable. In this work, the authors propose novel automated strategies to monitor the health of blowout preventers (BOPs) on offshore drilling rigs. Using physicsbased models, we demonstrate computational detection of pressure tests by identifying characteristic features in timeseries pressure data. After detection, we present a methodology for extracting features relevant for prognostic health monitoring, including pressure decay and hold durations. Augmenting these computational models with domain knowledge from BOP experts, we produce health indices (HIs) for the respective equipment as the output. In addition, we demonstrate the optimized enumeration and identification of possible pressure test plans for BOPs in different combinatorial configurations. By combining our novel detection approach with health indices and automated test planning, this work contributes to prediction and amelioration of well control equipment failures on offshore drilling rigs.
\end{abstract}

\section{INTRODUCTION}

A blowout preventer (BOP) is a safety-critical drilling rig component used primarily during exploration, development and workover phases of an oil and gas field. While a column

Se Un Park et al. This is an open-access article distributed under the terms of the Creative Commons Attribution 3.0 United States License, which permits unrestricted use, distribution, and reproduction in any medium, provided the original author and source are credited. of mud serves as primary means of controlling wellbore pressure during drilling, a BOP forms the final layer of protection that prevents a loss of well-control (LOWC) event from becoming a hazardous blowout. A study by Per Holland and the Bureau of Safety and Environmental Enforcement (2016) reports that approximately $60 \%$ of LOWC events occur during drilling operations.

A BOP is placed on top of wellhead with drilling equipment going through it into a well. Depending on operating context of LOWC event, a BOP must either isolate wellbore pressure by sealing around a tubular or drill pipe (Figure 1) or shear a tubular before isolating wellbore pressure. The task of isolating wellbore pressure from the drilling rig is accomplished by hydraulically energized elastomeric components referred to as 'packers' or 'seals' (Figure 2). For operational flexibility and redundancy, multiple BOPs are integrated vertically to form a 'BOP stack' (Figure 3).

A reliability study by American Bureau of Shipping and the Bureau of Safety and Environmental Enforcement (2013) indicates that approximately $50 \%$ of BOP stack failures are attributable to BOPs, while remaining are due to the BOP control system and other ancillary equipment. Due to its safety service, BOP failures do not become obvious until an operational demand is placed upon it.

Periodic tests of BOP stack allow drilling contractors to maintain and control the integrity of its functional service, as suggested by Martins, Cardoso, Tammela, Colombo, and Matos (2018). One type of such tests is a pressure test. A pressure test simulates a 'kick' (undesired increase in wellbore/annulus pressure) to assess ability of BOP packers to isolate anticipated well pressure. Because high pressure flowing fluid used in the test can erode metallic components and wear-out elastomeric components such as rubber side packers in rams in a BOP stack, typical aging effects of performance degradation over time are expected in 
mechanical properties of the system. The aging effects manifest in form of leaks of wellbore fluid past well-bore packers. The integrity of these components must be assessed regularly by simulating pressures on them. For a pressure test, the testing engineers temporarily stop regular rig functions and close a combination of annular preventers, rams, and valves to apply pressure within a BOP system and measure the pressure changes to assure that the BOP can hold the specified pressure. Towards this, BOP stack is instrumented with pressure and temperature $(\mathrm{P} / \mathrm{T})$ transducers exposed to wellbore.

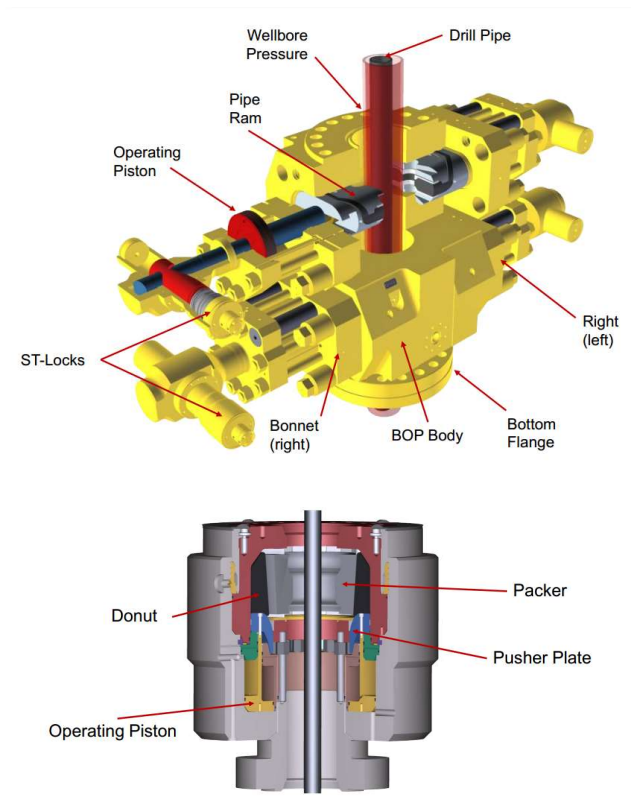

Figure 1. Blowout Preventers (BOP) that seal around drill pipe: Pipe Ram Preventer (above) and Annular Preventer. (below)

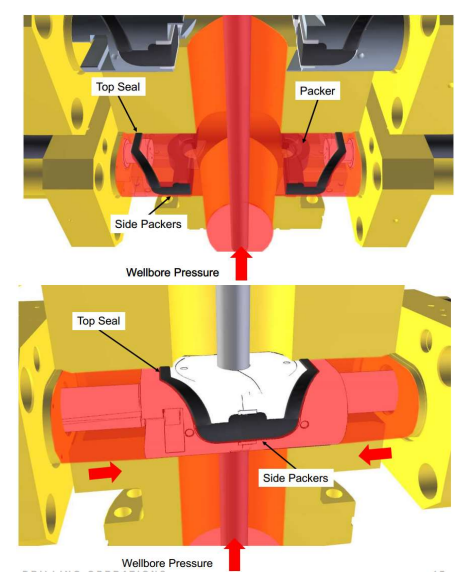

Figure 2. Wellbore Packers isolate pressure from the well in event of a 'kick'. Packers during normal operation (above) and Packers during test or 'kick'(below).
In this work, to detect any sign of failures upon pressure holding capacity in a component of a BOP stack, an automated solution within a prognostic health monitoring (PHM) framework is proposed, in accordance with Vachtsevanos, Lewis, Roemer, Hess, and Wu (2006), by using the above-mentioned pressure test data. A method that can identify and characterize pressure tests by searching the starting and finishing points of the test considering realistic physical constraints, is developed. Primary data consists of statuses of subsea BOP stack components and pressure as measured by a P/T sensor exposed to annulus of a BOP stack.

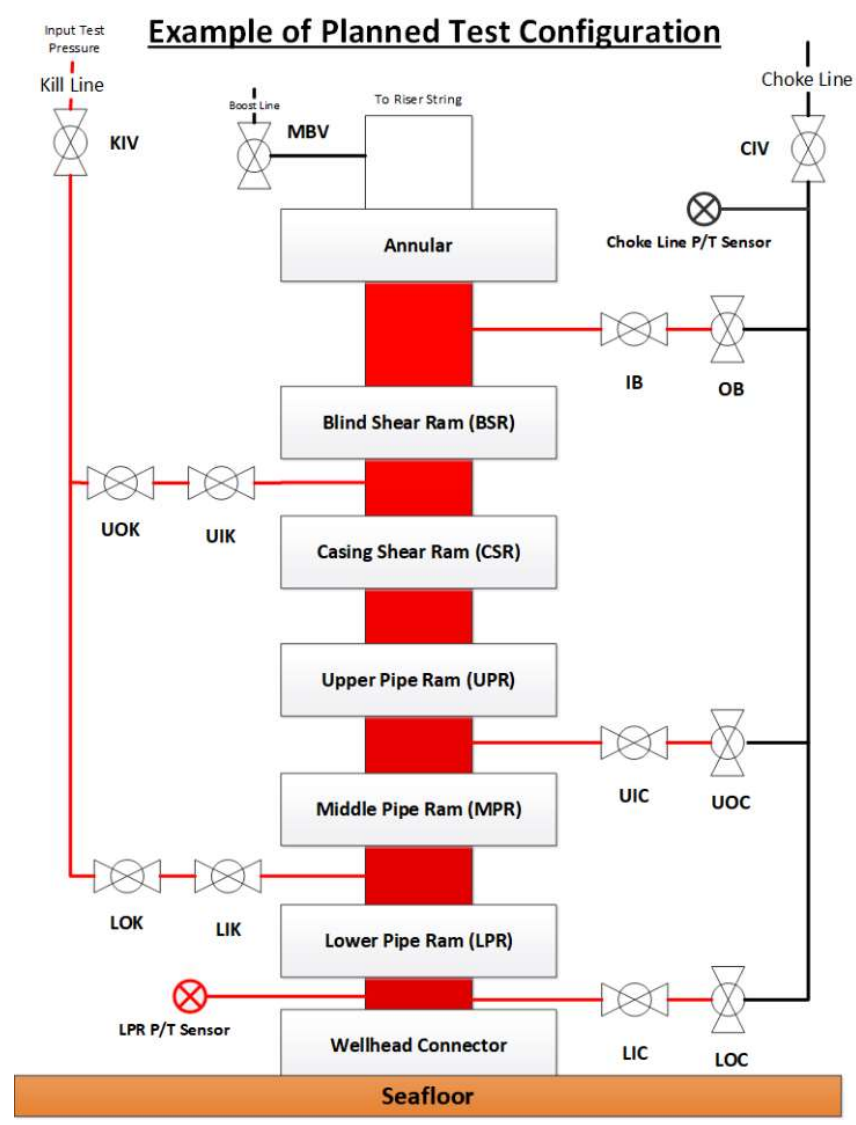

Figure 3. BOP Stack with 19x components: 1x Annular, 2x Shear Ram, 3x Pipe ram, 11x Gate valves and 2x Line isolation valves. Pressure \& Temperature Transducer mounted under Lower Pipe Ram (LPR) and under Choke Isolation valve (CIV).

The method also identifies which components of a BOP stack are being tested, because it comprises of numerous components such as annular BOPs, ram BOPs and fail-safe valves. This enables classification of test configuration and benchmarking of test performance for condition monitoring. Furthermore, a set of complete pressure tests must cover testing of all components by considering combinations of the closed, pressure-holding components. This needs to be planned to efficiently perform pressure tests of all 
components and avoid redundant tests. As testing often precludes further operational activities, test plan optimization is desirable to drilling contractors and operators to minimize non-productive time (NPT) on the rig.

With the goal of automating identification of tested components as well as for test planning, a simulator based on the specific structure of a BOP stack and physical constraints of each component is proposed. As a practical example, a pressured line may have multiple valves, e.g., inner/outer and upper/lower valves, and two sides of each valve should be tested to determine the health states of subcomponents; e.g., the right side of the subcomponent in upper inner choke valve. Also, pressured annular preventers and ram preventers typically have one directional resistance in pressure by design, i.e., pressure coming from the wellbore. Under the known states of BOP stack components, the simulator can determine lines and cavities that are pressurized, components tested, pressurized sides of tested components, and components whose status is irrelevant. The simulator discussed herein can be used in automation of test-planning.

\section{MethodS}

\subsection{Detection of Pressure Tests}

\subsubsection{Pressure Data Processing}

For the given BOP structure considered in this work, there are two $\mathrm{P} / \mathrm{T}$ (pressure and temperature) sensors: pressure sensor below the lower pipe ram (LPR) and above the wellhead connector, is referred to as LPR Pressure. The other sensor is located on the choke line. LPR Pressure data has better exposure to the pressure of the primary rams in the BOP stack because of the proximity and direct connectivity to the wellbore (For example, see Figure 3). While Maximum allowable working (rated) pressure of the BOP stack is 690 bar, the range of pressure sensors used is 0-1400 bar.

The pressure data from the LPR sensor is recorded with proper time stamps, but data was observed to be noisy or erroneous. The erroneous data, such as negative pressure or pressure values greater than the maximum working pressure of the BOP, can be removed. Other erroneous data are difficult to directly remove, but instead can be treated as noisy data points in time series data given pressure tests are characterized by shallow decay over 10-20 minutes duration. For denoising, a simple filtering approach is used.

Figure 4 illustrates one example of raw LPR Pressure data. Red and black dots indicate starting and ending points of high pressure tests, respectively. The integer numbers above the black dots show decay levels in bars from the starting points, which will be later used as a feature.

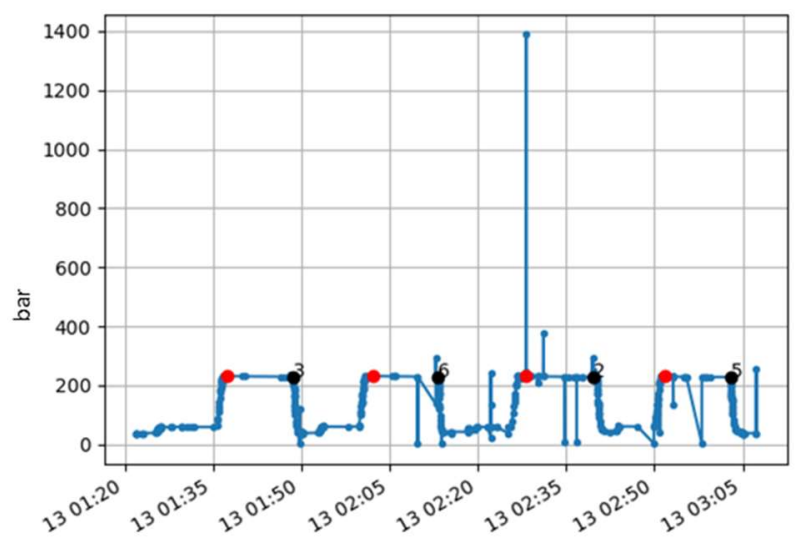

Figure 4. An example of raw BOP stack pressure test data.

(x-axis: time, y-axis: wellbore test pressure in bars)

Another issue of the pressure data may include resolution of pressure and sampling intervals or the combination of the two. This is especially noted during pressure tests where the differential pressures are small leading to sparse data sets. Irregularly sampled times series data is difficult to analyze or apply standard signal processing algorithms. The data is thus resampled or interpolated for further processing if needed.

\subsubsection{Identification of Characteristic Points of Pressure Tests}

The characteristic points of pressure tests include starting and ending points of pressure test hold-period. The test pressure pattern is mostly rectangular. This pattern has a seemingly flat top, but in fact has a small negative slope during the tests. The transitions of test-pressure (increase or decrease) are sudden and manifested as near-vertical lines in the time scale of hours as seen in Figure 4 and 5.

Limiting the prototypical pattern to a rectangular one, the first order differentials, as instantaneous or point-wise slopes, are ostensibly sufficient to identify the characteristic points. However, point-wise differentials are noisy with large variations because of the nature of the noise and data. Therefore, evaluation of the differentials over a larger fixed period-of-time stabilizes the value.

This approach is applied to identify the points in high pressure (HP) tests. Because most of the HP tests follow low pressure (LP) tests, the method is reapplied for the LP test identification. Figure 5 presents the detection results. We call the LP and HP, immediately following the previous LP, a unit pressure test. Each unit pressure test detected here corresponds to a unique configuration of BOP stack equipment. 


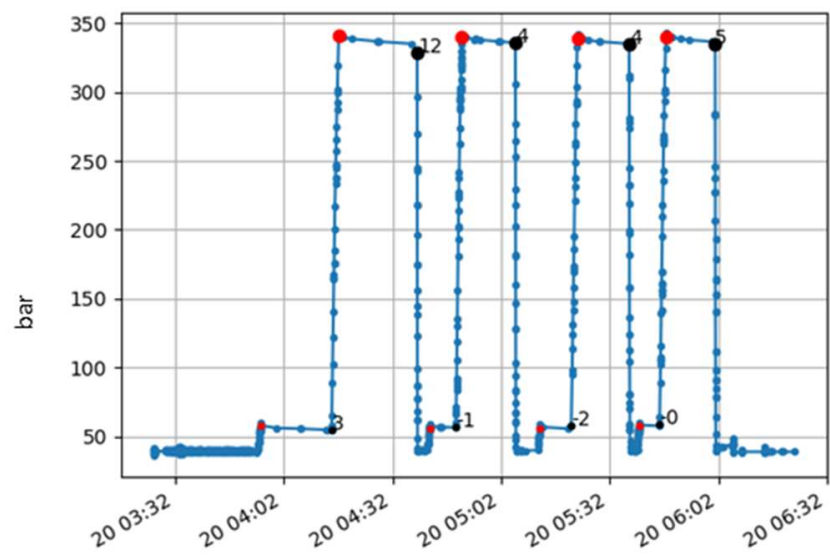

Figure 5. Detection and Identification of Low Pressure and High Pressure tests. (x-axis: time, y-axis: wellbore test pressure in bars)

\subsection{Feature Evaluation and Health Index}

Pressure test criteria identified by the American Petroleum Institute (API) standards (2012) or drilling contractors are evaluated as part of feature set. These features include hold duration between the starting and ending points of pressure tests and global decay (pressure difference from the ending to the starting points of tests). As a primary function of a BOP is to isolate pressure from well, wellbore pressure decay rate (rate of decrease in well bore pressure) lends itself well for a robust indicator to functional effectiveness of BOP wellbore packer.

Using the method and evaluated points, other features such as local decays defined by specific time-windows, the size of window in terms of time and the number of data points within the window, and statistics (mean, standard deviation) of data points within the window are evaluated.

Local decay and statistics are defined within a moving, sizevarying window as follows. Extend the window incrementally by covering a new data point in the time domain based upon a preselected datum and for each window repeatedly evaluate the local decay, slope, mean, standard variation, the number of data points within the window, and the window size in time. Other statistics, such as instantaneous slope or point-wise first order differentials, were experimentally attempted but ultimately not used because they show erratic, noisy behavior or are noninformative. Also, expected pressure ranges during tests seem wide enough for the data, i.e., the given HP test data fit within the specified range of $230-350$ bars, thus are noninformative.

Health indices are defined as the following. They represent how much the pressure test results conform to a minimum, required specification using the information of maximum allowed decay and minimum hold duration.

$$
\begin{gathered}
H I(s)=\max \left(T_{s}-s, 0\right) /\left|T_{s}\right|, \\
H I(d)=\max \left(T_{d}-d, 0\right) / d,
\end{gathered}
$$

Where $s$ is a slope (negative decay over time), $T_{s}$ is the specified threshold for the slope, $d$ is duration time of the pressure hold, and $T_{d}$ is the threshold for the duration time of the pressure hold. The overall health index is the sum of the slope and duration health indices, $H I(s)$ and $H I(d)$, respectively. Refer Figure 6 as examples of several health indices evaluated from different slopes and hold durations.

$$
H I=H I(s)+H I(d)
$$

The threshold values are typically given by various industrial regulatory guidelines, government agencies, or drilling contractors' prior knowledge. The defined health indices imply that for the perfect healthy state, $H I=0$ and is always non-negative and larger as the health becomes worse. The perfect healthy state covers the cases where the duration and slope of the pressure hold period are less than the corresponding threshold values. Note that the slopes and slope threshold are expected to be negative as the pressure tends to drop over time during the hold period of pressure test. The pressure drop can be simply modeled in either following way; if the pressure drop rate is proportional to the difference between the contained high pressure and outside pressure, i.e., $d P / d t=-k P(t)$, with a constant $k$, pressure $P$, and time $t$, the pressure has exponential decay, $P(t)=P_{0} e^{-k t}$, with the initial pressure $P_{0}$. If the pressure drop rate is constant over time due to the system control, i.e., $d P / d t=-k$, then the drop is simply linear, $P(t)=P_{0}-k t$. Under the resolution and range of data used here, the second model fits well and the pressure change model can be assumed linear.

In Figure 6, example health indicators are evaluated with a slope threshold of $1.3 \mathrm{bar} / \mathrm{minute}$ (absolute values are considered) and hold duration threshold of 12 minutes. If the absolute value of a slope gets larger than the threshold, HI increases from zero. If the hold duration is less than the threshold, then the corresponding $\mathrm{HI}$ is positive.

\subsection{Configurations}

Because every BOP component is not equipped with a pressure sensor, there is a need to tell which components are tested. For a specific BOP structure, to predict tested component, a manual compilation of all the possible test scenarios can be enumerated before testing and a dictionary of test configurations based on open/closed status of components can be built. This is feasible when the number of test cases is not large but requires automation as complexity increases. This automation can not only produce the dictionary of configurations, but also determine the tested components in real time. 

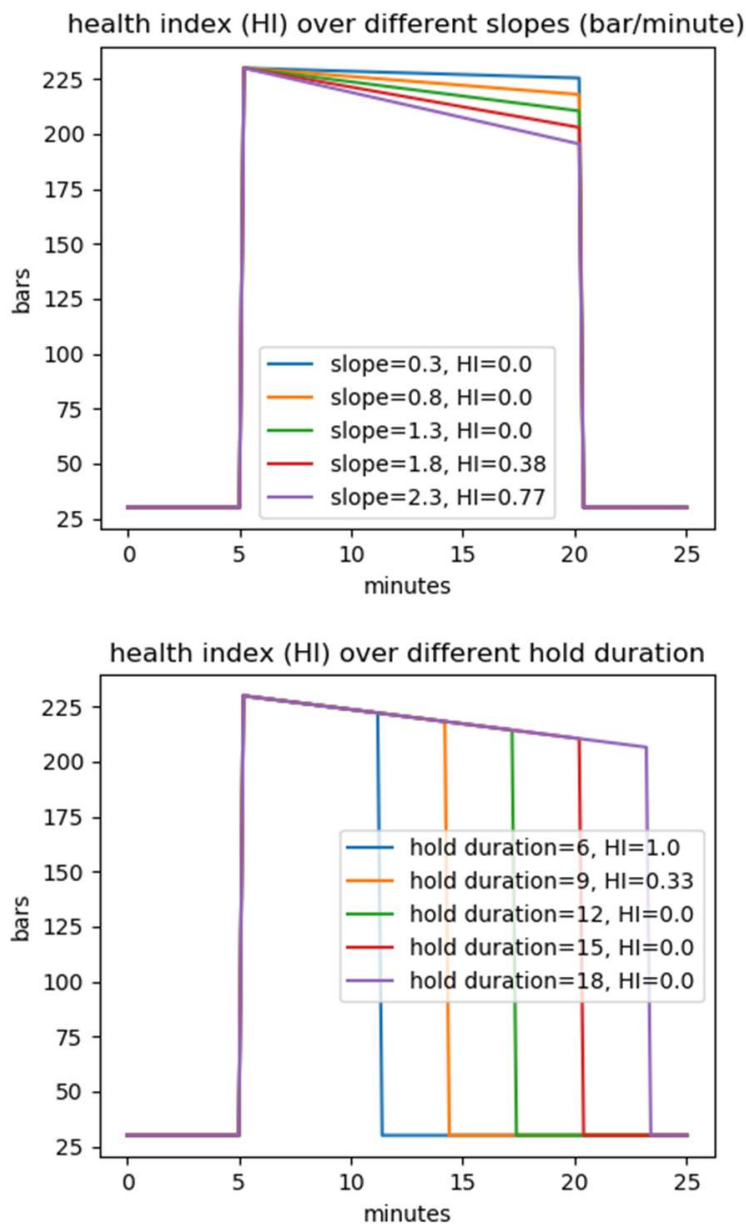

Figure 6. Pressure test patterns and evaluated health indices (HI): Health index over different slopes HI (s) (above) and Health index over different hold durations, HI(d) (below).

To identify tested components, states of all valves and preventers and pressure sources are read at the time of test execution and hold periods. For example, Figure 3 depicts an example pressure test configuration consisting of several components including Annular Preventer and the pressured line is kill line. Figure 7 lists open or close status of all components depicted in Figure 3.

By following pressure source to a blocked or closed component, pressured areas and tested parts are marked. In practice, there are more considerations such as the following:

- $\quad$ Pinpoint the tested side of valves, (outside or inside)

- at most one of the rams may be closed,

- if one of the paired (inner and outer) valves is closed and tested, the other may be open,

- annular preventers and rams may have one directional resistance in pressure, i.e., pressure coming from the wellbore,

- open/closed status of certain components may be irrelevant to a given test.

\begin{tabular}{|c|c|}
\hline \multicolumn{2}{|c|}{ Example Pressure Test Setup Configuration } \\
\hline Component & Valve State $(0=$ open, $1=$ closed $)$ \\
\hline Annular & 1 \\
\hline Blind Shear Ram & 0 \\
\hline Casing Shear Ram & 0 \\
\hline Upper Pipe Ram & 0 \\
\hline Middle Pipe Ram & 0 \\
\hline Lower Pipe Ram & 0 \\
\hline Upper Outer Choke (UOC) & 1 \\
\hline Upper Inner Choke (UIC) & 0 \\
\hline Lower Outer Choke (LOC) & 1 \\
\hline Lower Inner Choke (LIC) & 0 \\
\hline Choke Isolation Valve (CIV) & 0 \\
\hline Upper Outer Kill (UOK) & 0 \\
\hline Upper Inner Kill (UIK) & 0 \\
\hline Lower Outer Kill (LOK) & 0 \\
\hline Lower Inner Kill (LIK) & 0 \\
\hline Kill Isolation Valve (KIV) & 0 \\
\hline Outer Gas Bleed Valve (OB) & 1 \\
\hline Inner Gas Bleed Valve (IB) & 0 \\
\hline Mud Boost Valve & 1 \\
\hline
\end{tabular}

Figure 7. Status of BOP Stack Components involved in Example Pressure-test configuration depicted in Figure 3.

A BOP pressure test simulator under these considerations was designed. Table 1 presents a partial list of test configuration derived from the automated configuration identifier based on BOP stack depicted in Figure 3. A complete set of test configurations can help optimize the planning of BOP pressure tests. Configuration encoding determines which components are tested, not tested and ignorable regardless of their states ( 0 : open, $X$ : open or close, $\mathrm{T}$ : tested). The encoding is in the order of UAP, BSR, CSR, UPR, MPR, LPR, UOC, UIC, LOC, LIC, CLI, UOK, UIK, LOK, LIK, KLI, OB, IB, and MBV. The compiled dictionary output from the simulator matched that from manually evaluated list made by domain expert.

After a pressure test is finished, the extracted configuration will be looked up in the dictionary of planned configurations or inputted to the simulator to identify tested components and then we further generate feature values and health indices. If there is a discrepancy between extracted configurations and planned ones, e.g., a non-matching configuration, either the test may have been performed incorrectly, or our dictionary may not be complete and lacking some test cases. We record this case to $H I=-1$, to differentiate the normal nonnegative range of health index.

We note that blind spots may exist in identifying configurations, arising from lack of sensors for a given BOP structure. These blind spots relate to several configurations and unidentified sources. For instance, if the pressured sources are kill and choke lines and all outer valves are closed, then the increased pressure does not reach the sensor attached to LPR, thus there is no active pressure change recorded. 
Table 1. Partial Output of Automated Configuration Identifier for Example BOP Stack

\begin{tabular}{|c|c|}
\hline no. & configuration \\
\hline 1 & T00000T0T00XX000T0X \\
\hline 2 & 0000T0T0000 \\
\hline 3 & 00000 TT00 \\
\hline 4 & ООтоОХХ0०ОХХОT0TОХ \\
\hline & TOOOOXX0000T0T0TO \\
\hline
\end{tabular}

The benefit of the automation of configuration analysis includes easy extendibility to other complex BOP structures and efficient planning as a cost saving measure in rig operations. Using the automation, test engineers can perform grid-searches of all the cases or adopt Monte Carlo approaches to cover all the component tests while reducing the number of tests to the minimum, thus reducing cost and NPT involved in the tests.

\subsection{Health Decision}

Following evaluation of composite health index (based on decay and duration), test configuration is identified and observed health index is benchmarked to 'healthy' performance of that test configuration for a health decision.

Figure 8 depicts an overview of process flow involved in condition monitoring and health decision for BOP stack wellbore packers.

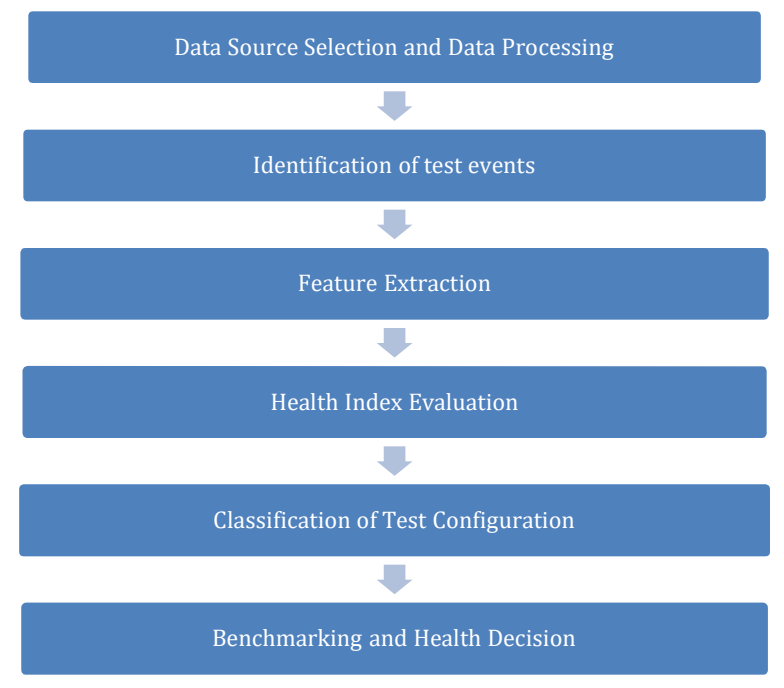

Figure 8. Condition Monitoring Process Flow for BOP Wellbore Packer.

\section{RESULTS}

\subsection{Application to a test well with one-year data}

Features and health indices based on pressure test data from an active drilling rig were evaluated. Condition monitoring method detailed in previous sections was applied to middle pipe ram (MPR) and upper annular preventer (UAP). While the rig did not experience any packer failures, it did report an opportune preventive replacement of UAP wellbore packer.

Refer to Figure 9 and Figure 10 for features for MPR. For the health index, due to its thresholding effect and global representation of a number per a pressure test, the obtained health indices were not as informative as features derived. Upon close inspection, the large HIs are caused by noisy data, which caused unstable and wrong estimation of hold-duration as denominator in the definition of HI, and not by degraded performance or large decay.

In generating local features, for the stability of evaluation, windows of at least three minutes and having at least three data points were considered. For the health index, the decayover-time threshold was set to $20 \mathrm{PSI} /$ minute and the holdduration threshold to 15 minutes.

Packer of UAP was changed in October 2017 and their features in different time scales are presented in Figure 11 and 12. There is no significant change in the trend of the presented features before and after an event in Oct 2017.

The proposed method was implemented for real-time monitoring of pressure tests and resultant health status. However, preventive maintenance of BOP Stack by the Drilling Contractor led to period of failure-free operations.

\subsection{Limitations and other promising approaches}

This study investigated features from LPR pressure data. Certain pressure tests of Choke \& Kill valves, cannot be detected by LPR pressure sensor. LPR pressure corresponding to these pressure tests showed the baseline pressure values without pressure change activities, leading to missed detection of pressure test. Pressure data from choke line can partially overcome this limitation. Investigation using the choke pressure data discovered several pressure tests that were not detected by using LPR data. However, addition of these tests does not seem to provide significant values because there are only few newly discovered tests using the choke pressure data. Also, almost the same information is obtained from the extracted features by using the choke pressure data regarding commonly detected tests. Moreover, considering the originally designed BOP function, holding pressure from the wellhead, and practical aspects of testing major BOP rams close to the wellhead, using LPR pressure data is sufficient for the purposes of this study.

Another limitation of the approach might be from physics of the failure of elastomer. The rubber material in rams and valves may be functioning acceptably well until it fails to resist against pressure in the event of sudden rupture or loss of the material. This type of sudden failure can be difficult to predict because it may not signal any gradual sign of wear or loss of integrity. If the remaining material integrity is the same as the new one after long use, measuring the volume loss of the material may better determine the remaining life of the seal. 


\section{Conclusion}

A framework for automated condition monitoring of BOP Stack wellbore packers based on pressure test data and physics of constraints is proposed. Methods for detection and identification of pressure tests, health index evaluation and identification of tested equipment are developed and implemented. Sensitivity of health indices to preventive maintenance was evaluated with field-data having no failures. In addition, an extension is proposed for optimization of BOP Stack pressure test plans through automated enumeration of test configurations.

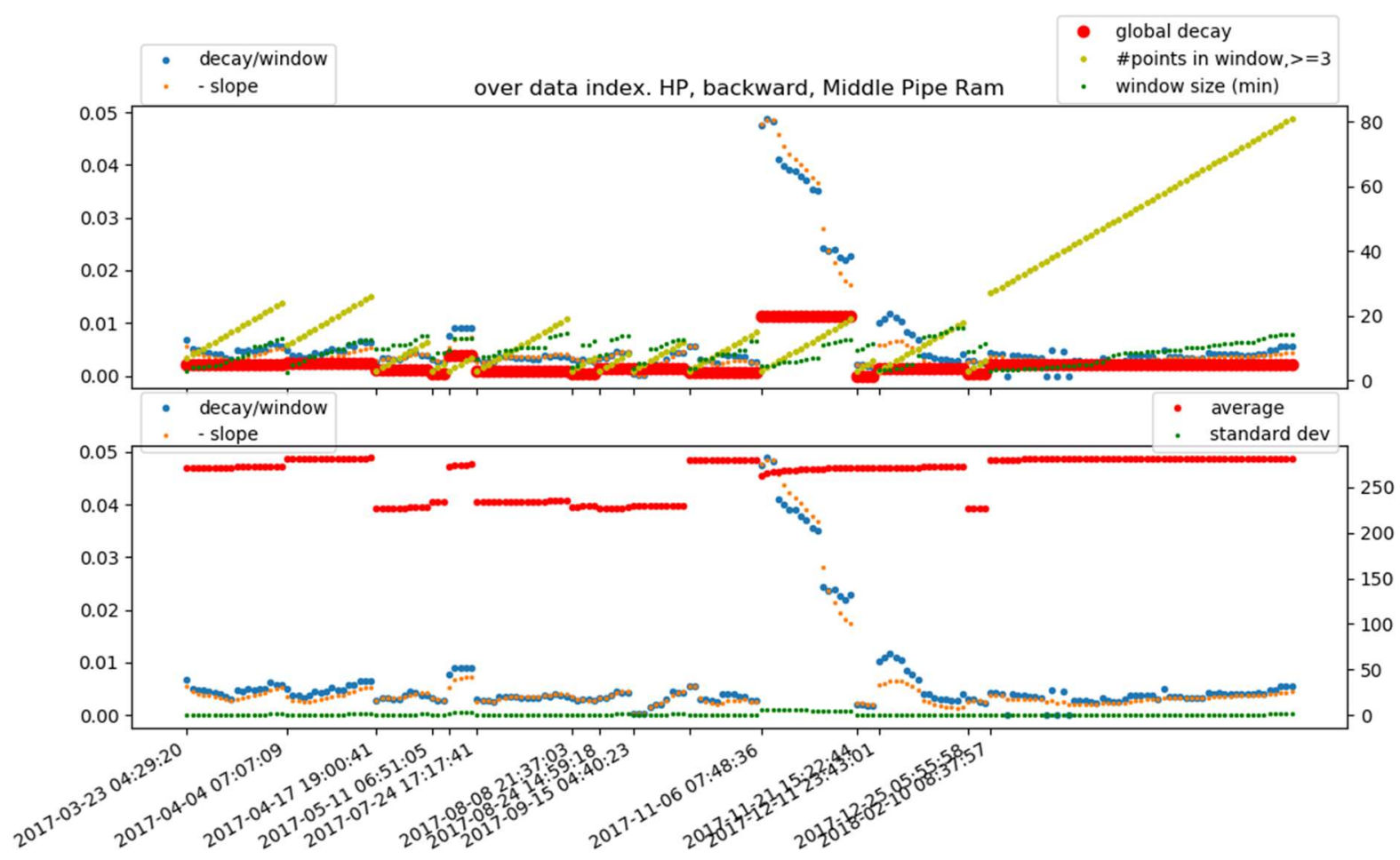

Figure 9. Features over index of data points for middle pipe ram. The time of pressure tests are shown in the x-axis of the bottom figure.

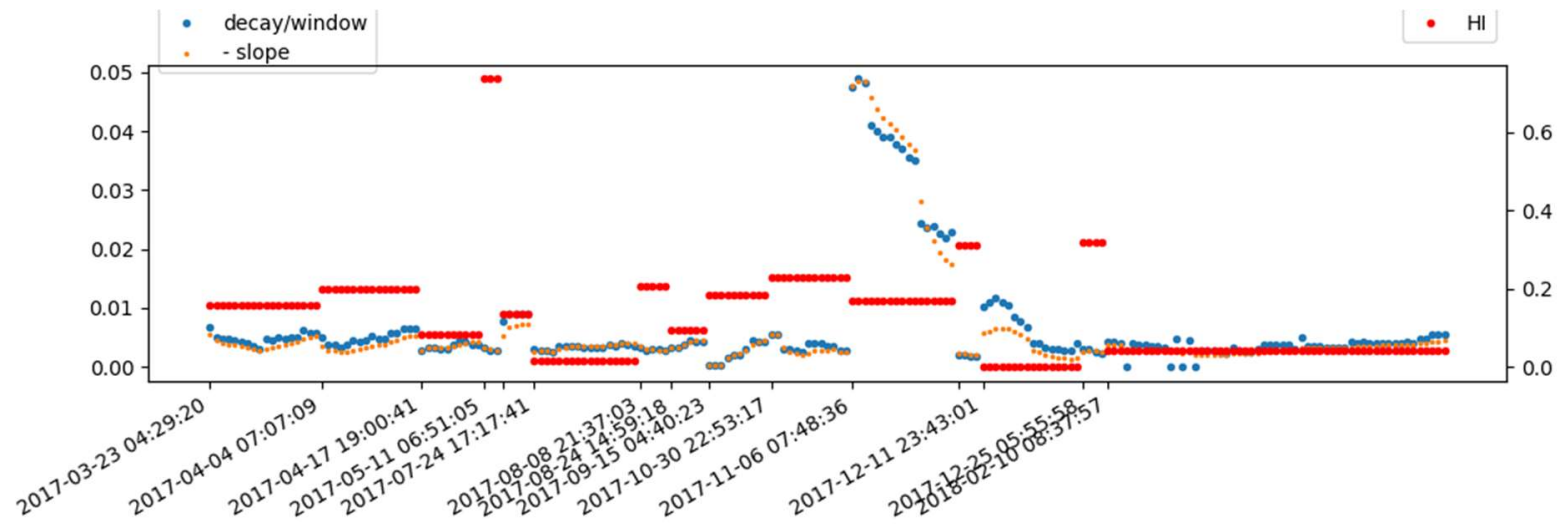

Figure 10. Health index (HI) over index of data points for middle pipe ram. 


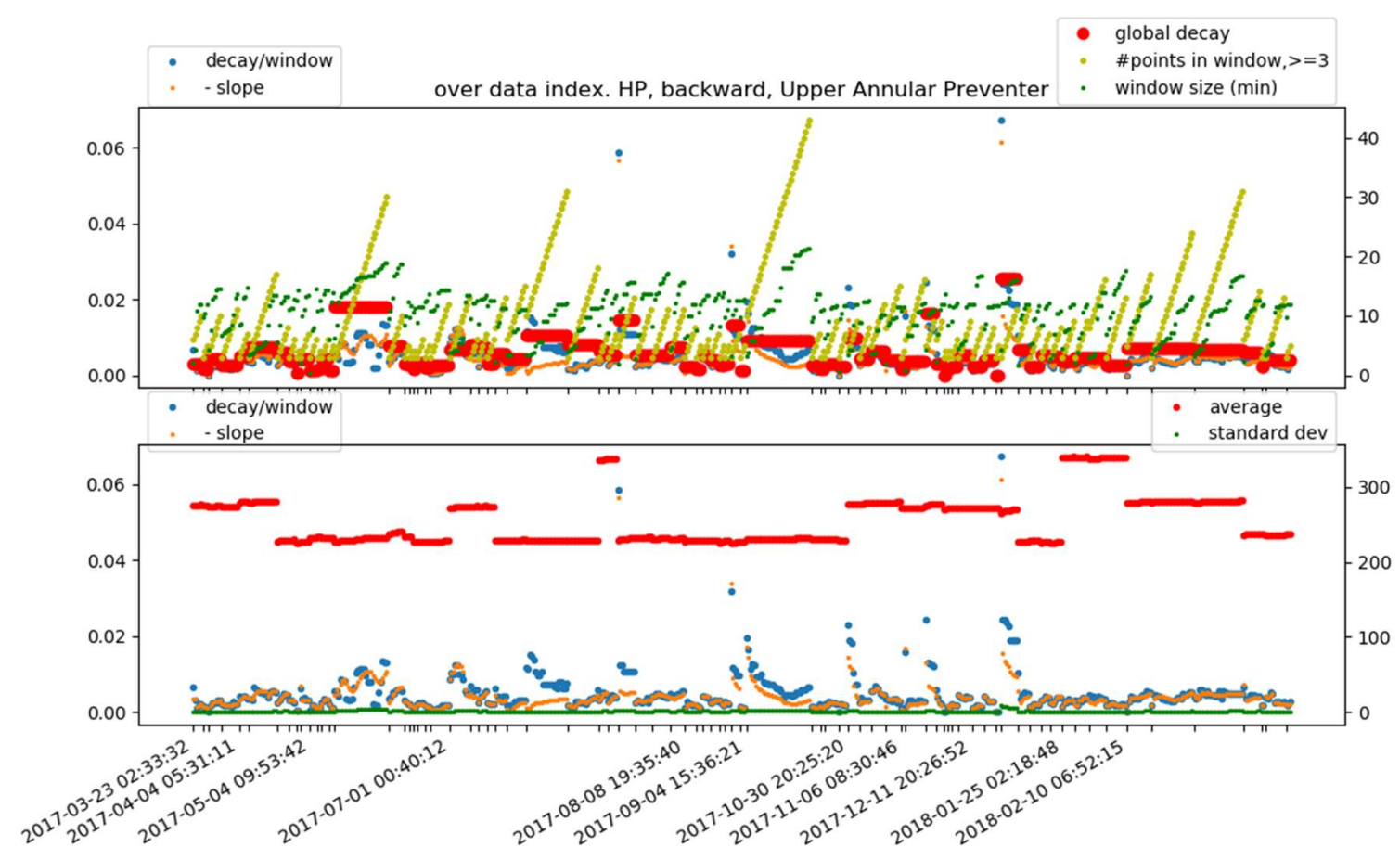

Figure 11. Features over index of data points for upper annular preventer. The drastic behavior on several occasions is due to data quality, not from actual outliers.

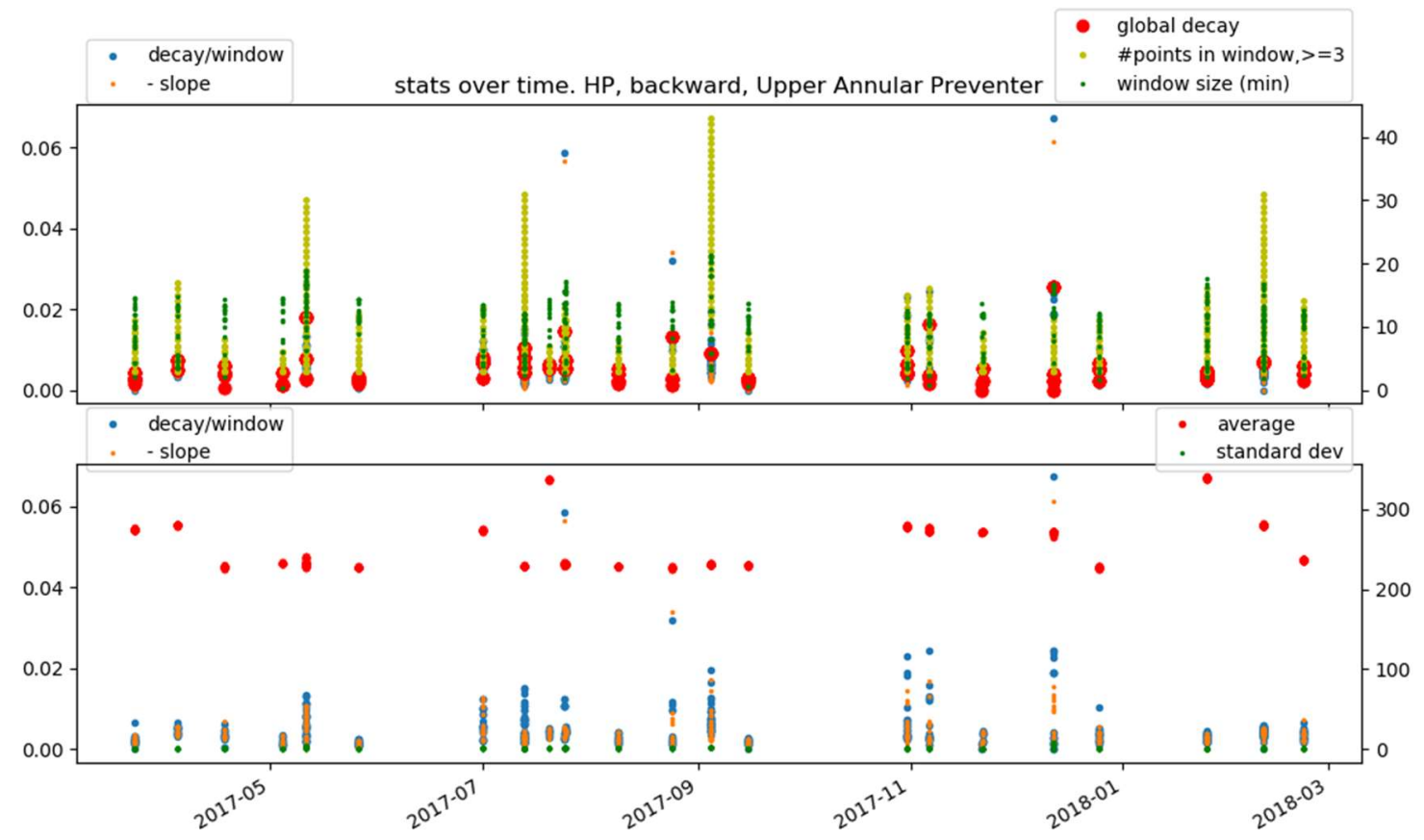

Figure 12. Features over time for upper annular preventer. (same information but in a different $\mathrm{x}$-axis scale from the previous figure) 


\section{REFERENCES}

American Bureau of Shipping \& the Bureau of Safety and Environmental Enforcement (2013), "BOP Failure Event and MIT Data Analysis for BSEE”, June 2013

American Petroleum Institute. (2012) “API Standard 53: Blowout Prevention Equipment Systems for Drilling Wells", Fourth Edition, November 11, 2012

Martins, F. B., Cardoso, R., Tammela, I., Colombo, D., \& Matos B. A. d., (2018). Applying CBM and PHM concepts with Reliability Approach for Blowout Preventer (BOP): A Literature Review. Brazilian Journal of Operations \& Production Management 15 (2018), pp $78-95$

Per Holland \& the Bureau of Safety and Environmental Enforcement (2016), "Loss of Well Control Occurrence and Size Estimators”, Sep 152016

Vachtsevanos, G., Lewis, F. L., Roemer, M., Hess, A., \& Wu, B. (2006). Intelligent fault diagnosis and prognosis for engineering system. Hoboken, NJ: John Wiley \& Sons, Inc

\section{BIOGRAPHIES}

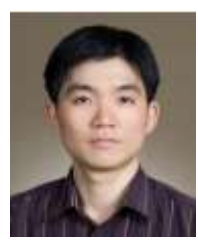

Se Un Park is a Senior Data Scientist with Schlumberger's Enterprise Solutions organization and received the Ph.D. degree in electrical engineering from the University of Michigan, Ann Arbor, MI, USA. His research interests include statistical signal processing, image processing, Bayesian estimation theory, and their applications to big data and prognostic health management. He co-authored three US patent applications and has contributed to numerous journal and conference papers as an author and reviewer, especially in the areas of image processing, machine learning, and neural networks.

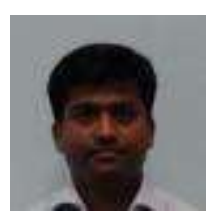

Rajesh Kumar Bade is a Senior Risk \& Reliability Quality Engineer on the Digital Solutions team of the Cameron Drilling Systems Product Line at Schlumberger. In this capacity, he works with product development and field service teams implementing datadriven solutions for real-time equipment performance insights and condition-based maintenance recommendations. He also advises Drilling Systems Risk \& Reliability team on subjects of Risk Assessments and Reliability Testing. He is a member of Technical Reference Group for IADC/IOGP Blowout Preventer (BOP) Reliability Joint Industry Project (JIP). He obtained his Master of Science in Mechanical Engineering from Texas A\&M University, College Station, TX in 2004.

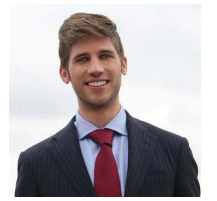

Daniel Barker is a Senior Control Systems Engineer in the new product development group within the Cameron Drilling Systems Product Line at Schlumberger. In this capacity, he has worked with teams developing next generation well control equipment. He has spearheaded initiatives and guided industry discussion on the implementation of systems modelling and simulation as a cost-effective tool for improving reliability and predicting dynamic performance of complex systems.

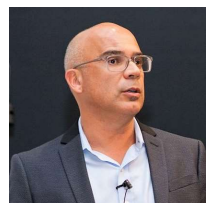

Daniel Edgardo Viassolo is a Principal and the Global PHM Analytics Manager with Schlumberger's Enterprise Solutions organization. Throughout his career, Daniel made strong contributions to the field of Industrial Asset Health Management and Controls across diverse applications domains - oil \& gas services, renewable energy, jet engines. Daniel co-authored 19 US patents (with several additional pending applications), more than 30 publications, and 1 book. He obtained his $\mathrm{PhD}$ from Purdue University. 